\title{
Encalhes de tartarugas marinhas no litoral sul de Pernambuco, Brasil
}

No nordeste do Brasil, os encalhes de tartarugas marinhas têm sido um instrumento importante de pesquisa para avaliação de impactos negativos que vem ocorrendo nas regiões costeiras. $O$ despejo de poluentes e resíduos sólidos no mar, a exploração dos recursos pesqueiros e a degradação de áreas costeiras tem sido as principais causas para o declínio de população de animais ameaçados como as tartarugas marinhas na costa do Brasil. A presente pesquisa teve como objetivo, analisar os encalhes de tartarugas marinhas no litoral do Ipojuca, Pernambuco, Brasil. Foi realizado um levantamento dos encalhes nas praias do Ipojuca, desde setembro de 2008 a junho de 2016, através de informações no banco de dados da Organização não Governamental Ecoassociados, bem como o monitoramento de $12 \mathrm{~km}$ dessas praias, entre os meses de setembro de 2015 a junho de 2016. Os animais encontrados mortos ou debilitados foram identificados e avaliados quanto a localização em relação a praia, sexo, faixa etária, estado de conservação da carcaça, evidência de interação antropogênica, presença de tumores, presença de anilhas e evidência de interação com outra fauna. Um total de 418 encalhe foram documentados, esses distribuídos em $16,98 \%$ de tartarugas não identificadas, $4,41 \%$ de tartaruga cabeçuda, 7,05\% de tartaruga de pente, $24,11 \%$ de tartaruga oliva, destacando a tartaruga verde (Chelonia mydas) como a espécie com maior número de registro $(64,4 \%)$ de encalhes na costa do lpojuca. Quanto à faixa etária, os juvenis ( $n=197)$ foram mais representativos que os adultos ( $n=143$ ) e fêmeas ( $n=113$ ) foram mais expressivas que os machos $(n=30)$. A praia de Merepe obteve maior número de encalhes ( $n=131)$. Cerca de $20 \%$ das carcaças estavam com a presença de cracas e 11,11\% apresentando tumores com indicativo de fibropapiloma, sendo exclusivo para C. mydas. Sendo assim, é necessário repensar em ações direcionadas para conservação das espécies, evidenciando a educação ambiental e a fiscalização como uma das principais medidas.

Palavras-chave: Ações Antrópicas; Fibropapilomatose; Pesca Incidental; Chelonia Mydas.

\section{Strandings of sea turtles in the south coast of Pernambuco, Brazil}

In the Northeast of Brasil, the strandings of sea turtles have been used as an important instrument of research to evaluate the negative impacts that is occurring in the coastal regions. The disposal of pollutants and debris into the sea, the explotation through fishing activities and the degradation of coastal areas have been the main causes to the decline of endangered animals populations as sea turtles in the Brazilian coast. The aim of this study was to analyze the strandings of sea turtles in the Ipojuca Coast, Pernambuco, Brasil. A survey of strandings was developed in the Ipojuca beaches, since September 2008 to June 2016 , through the informations from the database of Non Governmental Organization Ecoassociados, as well as the monitoring activity in $12 \mathrm{~km}$ of these beaches, from September of 2015 to June of 2016. The animals found dead or injured were identified and evaluated as the location in relation to the beach, gender, age group, conservation status of the carapace, evidence of antropogenic activity, presence of tumors, washers and evidence of interaction with other animals. A total of 418 strandings was recorded and distributed in $16,98 \%$ of non identified sea turtles, and $4,41 \%$ of loggerhead turtle (Caretta caretta), 7,05\% of hawksbill turtle (Eretmochelys imbricata), $24,11 \%$ of olive ridley turtle (Lepidochelys olivacea), and green turtle (Chelonia mydas) was the specie with major proportion $64,4 \%$ of strandings in Ipojuca coast. As the age group, the juveniles $(n=197)$ were more representative than the adults $(n=143)$. And about the gender, females ( $n=113)$ were more abundant than males $(n=30)$. About the beaches, Merepe beach had the major number of strandings $(n=131)$. About $20 \%$ of the carcass were present some barnacles and $11,11 \%$ presented tumors, representing fibropapillomatosis disease, exclusive to Chelonia mydas. Therefore, it is necessary to rethink in directed actions to conserve species, through an environmental education and oversight with the main measures.

Keywords: Antropic Actions; Fibropapillomatosis; Bycatch; Chelonia Mydas.

\section{Topic: Conservação da Biodiversidade}

Reviewed anonymously in the process of blind peer.
Received: $12 / 02 / 2019$

Approved: 28/03/2019
Karla Oliveira Silva

Universidade Federal de Pernambuco, Brasil

http://lattes.cnpq.br/9172026254489018

karlaconexao@gmail.com

Ednilza Maranhão dos Santos

Universidade Federal Rural de Pernambuco, Brasil

http://lattes.cnpq.br/5812920432455297

ednilzamaranhao@gmail.com

Thyara Noely Simões (iD)

Universidade Estadual de Santa Cruz, Brasil

http://lattes.cnpq.br/4718288776887692

http://orcid.org/0000-0001-8633-2169

thyara.noely@gmail.com
Arley Cândido da Silva

Universidade Norte do Paraná, Brasil

http://lattes.cnpq.br/6695684507238704

arley.ecoassociados@gmail.com
Referencing this:

SILVA, K. O.; SANTOS, E. M.; SIMÕES, T. N.; SILVA, A. C.. Encalhes de tartarugas marinhas no litoral sul de Pernambuco, Brasil. Revista Ibero Americana de Ciências Ambientais, v.10, n.2, p.53-64, 2019. DOI: http://doi.org/10.6008/CBPC2179-6858.2019.002.0006 


\section{INTRODUÇÃO}

As populações de tartarugas marinhas estão reduzindo no mundo inteiro e através da análise de encalhes têm se observado que a ação humana está ligada de maneira direta ou indireta nesse contexto (WYNEKEN et al., 1988). As causas antropogênicas podem ser exemplificadas pela captura incidental em redes, anzóis de pesca e 'bycatch', poluição marinha através de resíduos sólidos, fibropapilomatose, acidentes com embarcações, roubo de ovos e o consumo da carne (WYNEKEN et al., 1988; BUGONI et al., 2001; SILVA et al., 2010; CLUKEY et al., 2017). A interação de tartarugas marinhas com a pesca de espinhel é relatada por Sales et al. (2008) como uma problemática quanto à conservação desses animais dentro da Zona Exclusiva Econômica (ZEE) no Brasil, como também destacam que essa pesca tem sido feita diretamente em locais de forrageamento de espécies como L. olivacea e C. caretta, desencadeando altos índices de mortalidade.

De acordo com Epperly et al. (1996), os encalhes de tartarugas marinhas têm sido uma importante ferramenta de identificação dos principais impactos ambientais ocorridos no mundo. Segundo Camacho et al. (2013), os encalhes registrados na Espanha foram relevantes para apontar como o derramamento de óleo causou a morte de vários espécimes. Por outro lado, Vélez-Rubio et al. (2013), evidenciaram na costa do Uruguai que a localização e distribuição dos encalhes estavam diretamente ligados ao esforço de pesca junto a sazonalidade, como também relataram que o lixo antropogênico tem sido um problema para as espécies C. mydas e C. caretta, pois esses animais utilizam a costa desse país como área de forrageamento, alimentando-se de uma grande quantidade de resíduos sólidos.

No Brasil, os estudos de encalhes que foram realizados por Silva et al. (2012) e Poli et al. (2014) evidenciaram as diversas causas das mortes desses animais, como a pesca incidental, as grandes embarcações e a poluição na zona costeira. No estado do Rio grande do Sul foram registrados 648 encalhes de outubro de 2007 a dezembro de 2010, com registros de C. caretta $(n=284)$, C. mydas $(n=278), D$. coriacea ( $n=27$ ) e 51 indivíduos não identificados (SCHERER et al., 2014). Na região Nordeste, os estudos de encalhes de tartarugas marinhas são mais frequentes nos estados da Bahia, Paraíba e Sergipe (MACEDO et al., 2011; POLI et al., 2014; SILVA et al., 2010). Nessa região o número de encalhe variou entre 45 a 655 dependendo do estudo, pois alguns estudos envolviam apenas uma temporada de desova de tartarugas até anos de registros de encalhes. Na Paraíba, por exemplo, foram registrados 124 encalhes em apenas uma temporada de agosto de 2009 a julho de 2010, com maior representação de C. mydas, sendo a maioria das carcaças identificadas no estado de decomposição avançado (POLI et al., 2014).

No Litoral do Ipojuca há a ocorrência de quatro espécies de tartarugas marinhas no período reprodutivo, são elas: Eretmochelys imbricata (Linnaeus, 1766), Chelonia mydas (LINNAEUS, 1758), Caretta caretta (LINNAEUS, 1758), Lepidochelys olivacea (ESCHSCHOLTZ, 1829), as quais encontram-se nas categorias criticamente ameaça de extinção (CR), em perigo de extinção (ER) e vulnerável (VU) respectivamente (IUCN 2018). Segundo Guimarães et al. (2011), apesar de se ter a ocorrência dessas espécies ameaçadas de extinção, os danos que elas têm sofrido nessa região ainda não foram mensurados, evidenciando a 
necessidade de um diagnóstico atual, pois trata-se de uma região com intensa atividade de nidificação desses animais.

Dada à importância que os registros de encalhe podem oferecer como suporte ao manejo e conservação de tartarugas marinhas no litoral Sul de Pernambuco, Brasil, este estudo teve como objetivo analisar a frequência de encalhes de tartarugas marinhas entre os anos de 2008 e 2016, evidenciando padrões sazonais, bem como analisar quanto à riqueza, abundância, biometria do casco, sexo e faixa etária, situação da carcaça, vestígios de resíduos, tumores, injúrias e presença de cracas durante a temporada 2015/2016. E assim, promover um diagnóstico que venha contribuir para ações voltadas à conservação de tartarugas marinhas no litoral brasileiro.

\section{MATERIAIS E MÉTODOS}

\section{Área de estudo}

O presente estudo foi realizado na região Nordeste do Brasil, nas praias do município do Ipojuca, situado na mesorregião metropolitana e na microrregião de Suape do Estado de Pernambuco, Brasil $\left(08^{\circ} 24^{\prime} 25^{\prime \prime} \mathrm{S} ; 35^{\circ} 03^{\prime} 45^{\prime \prime} \mathrm{W}\right.$ ) (Figura 1). O litoral apresenta $32 \mathrm{~km}$ de extensão do qual apenas $12 \mathrm{~km}$ de costa é monitorado diariamente, que compreende as praias de Muro Alto, Cupe, Merepe, Porto de Galinhas, Maracaípe e o Pontal de Maracaípe (MOURA et al., 2012).

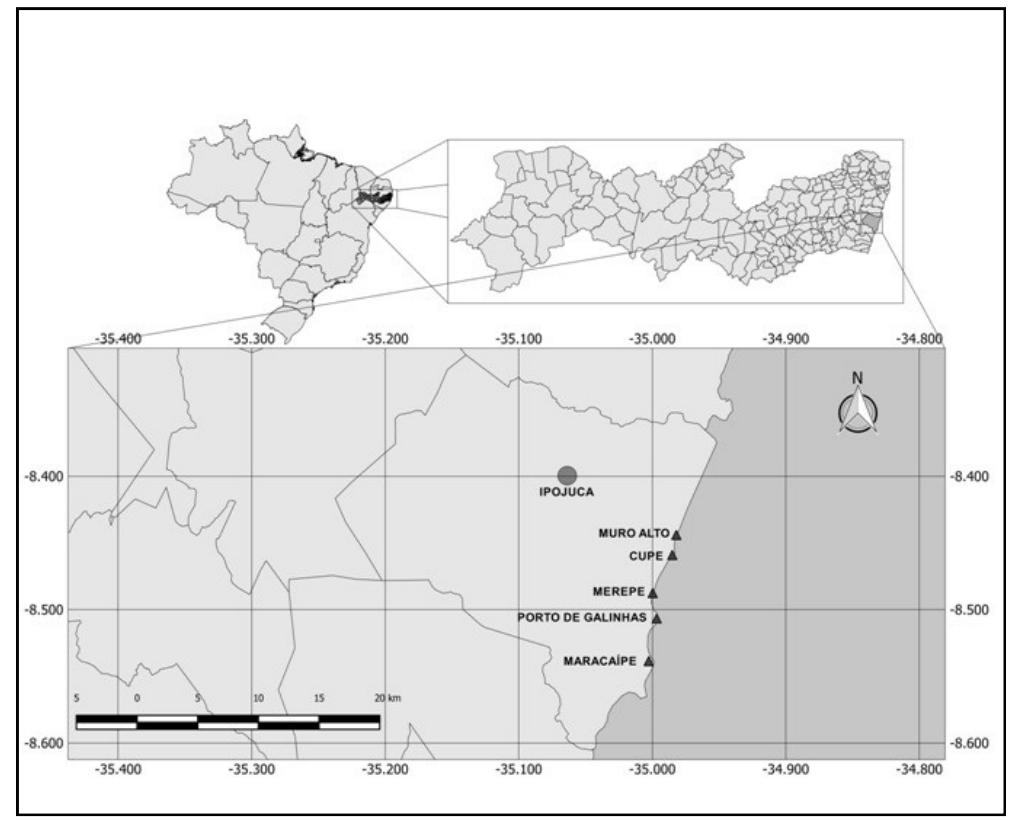

Figura 1: Mapa de localização da área estudada, evidenciando as praias onde há registro de encalhes de tartarugas marinhas e o monitoramento pela ONG Ecoassociados.

\section{Procedimentos metodológicos}

Os encalhes foram notificados em parceria com a ONG Ecoassociados, como também com a cooperação da comunidade local (contatando o número da Ecoassociados). Neste estudo, inicialmente foram computados e analisados à frequência e a espécie do encalhe de dados armazenados em relatórios da ONG 
Ecoassociados entre os meses de setembro de 2008 até junho 2016 e então foi realizada a análise detalhada dos encalhes ocorridos no período de 10 meses de setembro de 2015 a junho de 2016.

Para os registros relacionados à temporada 2015/2016, os indivíduos mortos foram identificados, georeferenciados através de um GPS, anotados em planilha o sexo, faixa etária, estado de conservação da carcaça, evidência de interação antropogênica, presença de tumores e evidência de interação com outra fauna. O estado de conservação da carcaça foi dividido em três categorias: a) sem evidência de decomposição, b) decomposição moderada e c) decomposição avançada (POLI et al., 2014). Para a classificação de juvenis e adultos de acordo com a metodologia descrita por Poli et al. (2014), onde foi obtido o comprimento curvilíneo da carapaça (CCC) e a largura curvilínea da carapaça (LCC), utilizando fita métrica maleável com precisão de 0,1cm (WYNEKEN et al., 2001), e para identificação das espécies foram utilizados características morfológicas, sem a utilização de necropsia. Após a coleta dos dados, os indivíduos foram enterrados na praia. No caso de animais encalhados vivos, estes foram encaminhados para reabilitação no projeto da ONG Ecoassociados. E para os animais vivos e mortos foi anotada a data para posterior verificação de sazonalidade. Além disso, também foram coletados dados abióticos, como médias de temperatura máxima e mínima, pluviosidade e umidade relativa através do Instituto de meteorologia do Recife na estação mais próxima, a Estação do Curado/PE, ao local de estudo.

\section{Análise de dados}

Para os dados gerais (número de encalhes, sexo, faixa etária e biometria do animal) foi utilizada a estatística descritiva, com valores numéricos, média e desvio padrão. Em seguida, testou-se a normalidade aplicando o teste Kolmogorov-smirnov e Shapiro Wilk's, posteriormente, utilizou-se a correlação de Spearman $\left(r_{s}\right)$ Rank Order para correlacionar o número de encalhes mensal com fatores abióticos como temperatura, umidade relativa do ar e pluviosidade.

Para testar diferenças entre as médias quanto ao número de encalhes entre os meses e ano foi utilizado o test Kruskal-wallis, ambos foram verificados através do programa Statistica 8.0 implementada pelo programa Statistica versão 7.0 (STATSOFT, 2013). E para analisar a significância da razão sexual e diferenças entre jovens e adultos e entre o número de encalhes e espécie foi aplicado o teste do Quiquadrado ${ }^{\left({ }^{x 2}\right.}$ ), disponível no programa BioEstat 5.0 (AYRES et al., 2007). Para todos os testes foi considerado significante quando o $p \leq 0,05$. A distribuição dos encalhes durante a temporada 2015-2016 foi feita através da plotagem das localizações em mapas, utilizando o programa Arcview, visando identificar áreas de maior concentração de encalhes.

\section{RESULTADOS}

Foi registrado um total de 418 encalhes de tartarugas entre os anos 2008 a 2016, desses, 78 indivíduos não foram identificados devido ao estágio muito avançado de decomposição. Os encalhes foram distribuídos entre 24 espécimes de E. imbricata, 15 de $C$. caretta, 82 de L. olivacea e 219 espécimes de $C$. mydas. Sendo as diferenças significativas entre os números de encalhes e as espécies $(p<0.000)$. A maioria 
dos indivíduos eram juvenis ( $n=197)$, dos adultos ( $n=143$ ) destacou-se 113 indivíduos fêmeas encalhados mortos (Tabela 1), com uma representação de $79,02 \%$. Houve diferença significante entre os números de jovens e adultos encalhados ( $p=0,0043)$, e entre machos e fêmeas $(p<0,000)$.

Em relação ao número total de encalhes, a média foi de 39,75 encalhes por ano, todavia para a última temporada investigada entre 2015 e 2016, o número foi de 54 casos de encalhes nos 12 km de praias, o que contabilizou um total de 53 espécimes mortos e um reabilitado, esse último devolvido ao mar. O único animal, da espécie $C$. mydas, encalhado vivo tinha evidências de rede de pesca na nadadeira direita anterior, que por problemas de circulação sanguínea teve que ser amputada. Os vestígios antropogênicos não foram representativos nos meses de setembro de 2015 a junho de 2016, com apenas um animal com restos de rede em uma das nadadeiras. Pois as carcaças foram observadas apenas externamente, não sendo evidente a interação antropogênica.

Quanto ao tamanho dos animais encalhados foram considerados os intervalos de comprimento curvilíneo da carapaça distribuídos para cada espécie, o que variou entre $80-120 \mathrm{~cm}$ (fêmeas adultas) e 28$77 \mathrm{~cm}$ (juvenis/ subadultos) para C. mydas, de 79-107 cm (fêmeas adultas) e 65-75 cm (juvenis/ subadultos) para C. caretta, entre $61-77 \mathrm{~cm}$ (fêmeas adultas) e $58 \mathrm{~cm}$ para L. olivacea e entre $84-99 \mathrm{~cm}$ (fêmeas adultas) e $36-68 \mathrm{~cm}$ (juvenis) para E. imbricata. As maiores médias foram para fêmeas de $C$. mydas e macho de $C$. caretta (Tabela 1), nos anos monitorados pela ONG Ecoassociados de setembro de 2008 a maio de 2015. Não houve diferenças significativas de tamanho entre o sexo das espécies registradas.

Tabela 1: Média e desvio padrão (DP) relacionados à idade e sexo das tartarugas marinhas encalhadas no litoral do Ipojuca/PE entre 2008 a 2016. Número de indivíduos (N), comprimento curvilíneo da carapaça (CCC) e largura curvilínea da carapaça (LCC)

\begin{tabular}{|c|c|c|c|c|c|c|c|}
\hline \multirow[t]{3}{*}{ ESPÉCIES } & & \multicolumn{4}{|l|}{ ADULTO } & \multirow{2}{*}{\multicolumn{2}{|c|}{ JOVEM }} \\
\hline & & \multicolumn{2}{|l|}{ FÊMEA } & \multicolumn{2}{|l|}{ MACHO } & & \\
\hline & & média $\pm \mathrm{DP}$ & $\mathbf{N}$ & média $\pm \mathrm{DP}$ & $\mathbf{N}$ & média $\pm \mathrm{DP}$ & $\mathbf{N}$ \\
\hline \multirow[t]{2}{*}{ C. mydas $(n=219)$} & $C C C$ & $97,85 \pm 12,69$ & \multirow[t]{2}{*}{$(n=32)$} & $93,75 \pm 19,48$ & \multirow[t]{2}{*}{$(n=4)$} & $52,84 \pm 12,69$ & \multirow[t]{2}{*}{$(n=183)$} \\
\hline & $L C C$ & $90,26 \pm 10,32$ & & $89,25 \pm 17,59$ & & $48,45 \pm 12,73$ & \\
\hline \multirow{2}{*}{$\begin{array}{l}\text { C. caretta } \\
(n=15)\end{array}$} & $C C C$ & $95,88 \pm 10,38$ & \multirow[t]{2}{*}{$(n=9)$} & $97,66 \pm 11,01$ & \multirow[t]{2}{*}{$(n=3)$} & $71,5 \pm 5,67$ & \multirow[t]{2}{*}{$(n=3)$} \\
\hline & $L C C$ & $89,77 \pm 10,10$ & & $90 \pm 11,35$ & & $69,33 \pm 6,50$ & \\
\hline \multirow{2}{*}{$\begin{array}{l}\text { L. olivacea } \\
(n=82)\end{array}$} & $C C C$ & $66,31 \pm 4,02$ & \multirow[t]{2}{*}{$(n=61)$} & $67,4 \pm 2,96$ & \multirow[t]{2}{*}{$(n=20)$} & & \multirow[t]{2}{*}{$(n=1)$} \\
\hline & $L C C$ & $65,86 \pm 3,69$ & & $67,4 \pm 2,96$ & & $67 \pm 2,44$ & \\
\hline \multirow{2}{*}{$\begin{array}{l}\text { E. imbricata } \\
(n=24)\end{array}$} & CCC & $88,36 \pm 10,26$ & \multirow[t]{2}{*}{$(n=11)$} & $89 \pm 9,53$ & \multirow[t]{2}{*}{$(n=3)$} & $47,3 \pm 11,40$ & \multirow[t]{2}{*}{$(n=10)$} \\
\hline & $L C C$ & $80,72 \pm 8,75$ & & $79 \pm 14,79$ & & $50,8 \pm 11,42$ & \\
\hline
\end{tabular}

Houve maiores números de encalhes entre os meses mais quentes (janeiro a abril e setembro a dezembro) e com os menores índices pluviométricos (novembro/2012 com 9,9 $\mathrm{m}^{3}$ ) nos anos de 2008 a 2016, o mês de fevereiro obteve um número maior de encalhe ( $n=14$ ) (Figura $2 \mathrm{~A}, \mathrm{~B}$ ), e o ano com maior número de encalhe foi 2013 ( $n=67$ ) (Figura 2 B). Todavia durante a temporada reprodutiva, monitorada entre 20152016, verificou-se que o mês de outubro obteve um maior número de encalhes (Figura 2 C). Ao correlacionar os números de encalhes e os parâmetros abióticos só foram possíveis obter valores significantes entre número total de encalhes e umidade relativa do ar $(p=0,0091)$, número de indivíduos de $C$. mydas com a pluviosidade e a umidade relativa $(p=0,0084)$ e o número de registros de $L$. olivacea com a umidade relativa do $\operatorname{ar}(p=0,0018)$. 


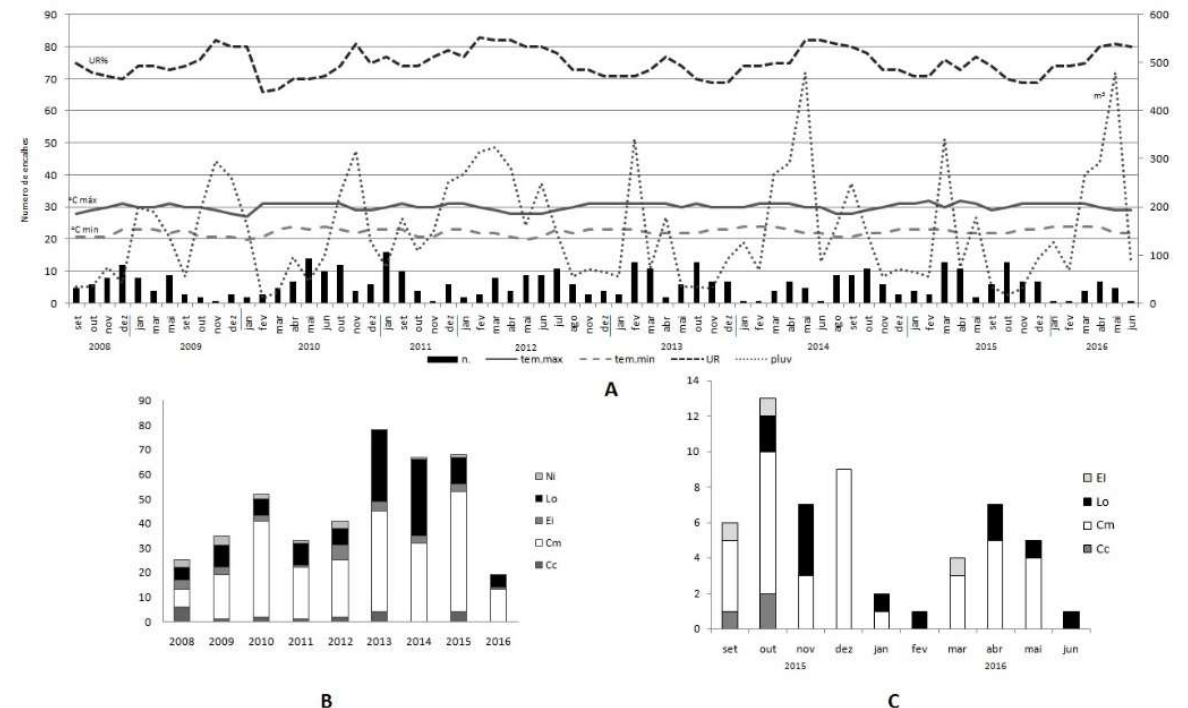

Figura 2: Distribuição mensal de encalhe de tartarugas marinhas no litoral do Ipojuca/PE entre os anos de 2008 a 2016 e parâmetros abióticos como umidade relativa do ar (UR), temperatura máxima (temp. máx) e mínima (temp. mín) e pluviosidade (pluv) (A), distribuição dos encalhes com as quatro espécies de tartarugas e os indivíduos não identificados (NI) (B), distribuição dos encalhes das tartarugas durante os meses relacionado à última temporada, 2015-2016 (C).

Quanto ao registro de encalhes de tartarugas marinhas e seu estado de decomposição, observou-se um maior número de carcaças em decomposição avançada nas espécies $C$. mydas e $L$. olivaceae. As espécies E. imbricata e $C$. caretta foram as menos representativas, demonstrando assim alguns poucos indivíduos em decomposição avançada, moderada e outros sem evidência de decomposição (Figura 3).

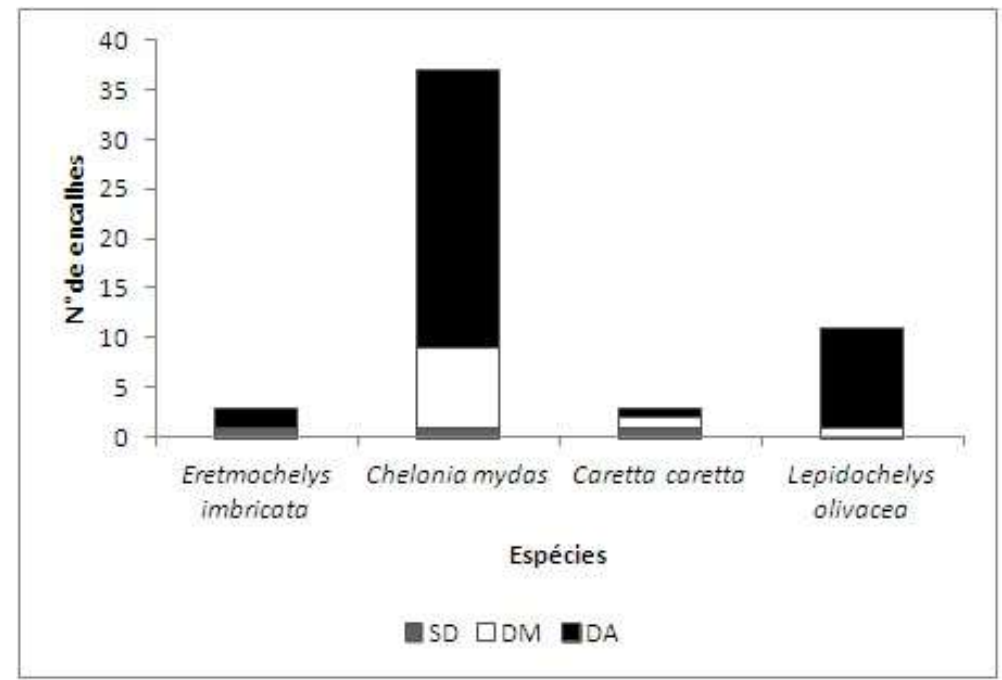

Figura 3: Registro de encalhes de tartarugas marinhas por espécie e estágio de decomposição entre 2015 e 2016 no litoral do Ipojuca/PE. Os estágios de decomposição foram divididos em: sem decomposição - SD (cinza), decomposição moderada - DM (branco) e decomposição avançada - DA (preto).

Durante os meses de setembro/2015 a junho/2016 foram contabilizados 54 pontos de encalhe de tartarugas nas praias (Figura 4), destacando-se a praia de Merepe com 17 registros (Figura 4 A). A espécie com maior representatividade foi a $C$. mydas $(n=37)$, tendo registro de encalhes em exatamente todas as praias estudadas (Figura 4 C). A espécie L. olivacea foi a segunda com maior representatividade ( $n=11)$. E as duas espécies que tiveram a menor taxa de encalhes foi a $E$. imbricata e a $C$. caretta, sendo cada uma registrada em apenas duas praias (Figura 4 B). 


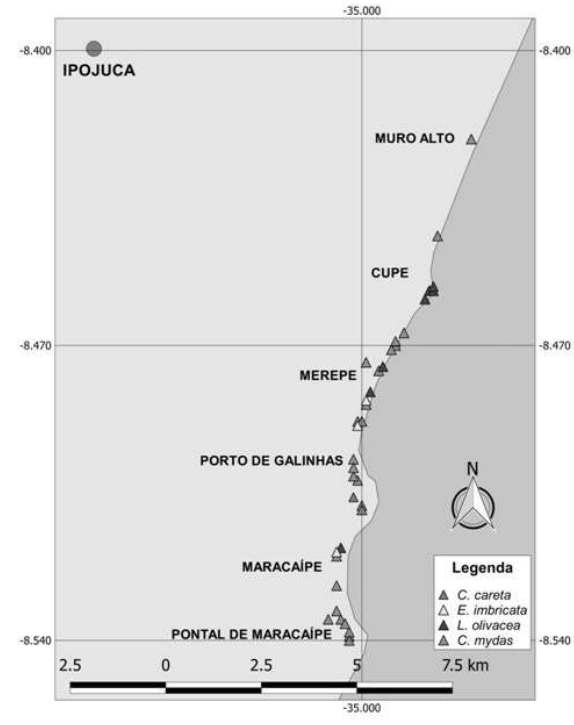

A
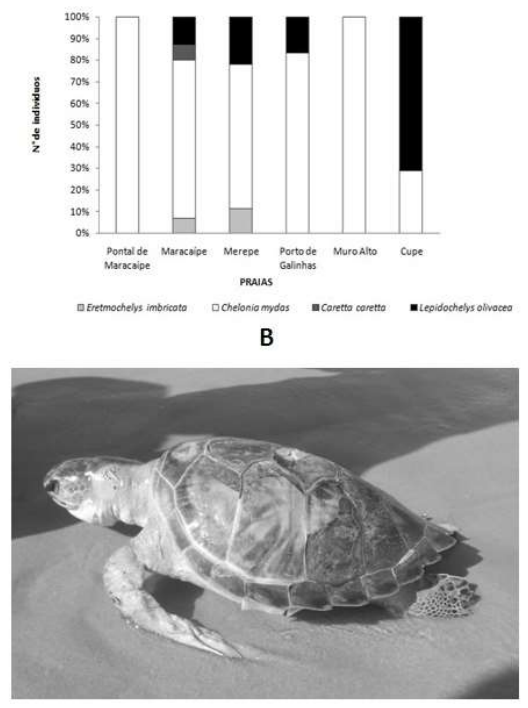

C

Figura 4: Distribuição das espécies de tartarugas marinhas encalhadas ao longo da litoral do Ipojuca no período de setembro de 2015 a junho de 2016 (A). Gráfico com indivíduos distribuídos entre as praias de Ipojuca (B). Imagem de

tartaruga verde juvenil encalhada na praia de Merepe (C), sendo esta a espécie de maior número de encalhes no litoral do Ipojuca.

Foi observado a presença e ausência de cracas nas tartarugas marinhas encalhadas no litoral do Ipojuca/PE. A espécie $C$. mydas teve o maior número de encalhes com presença de cracas ( $n=16,66 \%)$, e a espécie $E$. imbricata teve uma representação de 3,70\%, e as demais espécies obtiveram uma representação de 1,85\% desse crustáceo. Os tumores só foram observados na espécie $C$. mydas com presença em 11,11\% do total de encalhes (Figura 5).

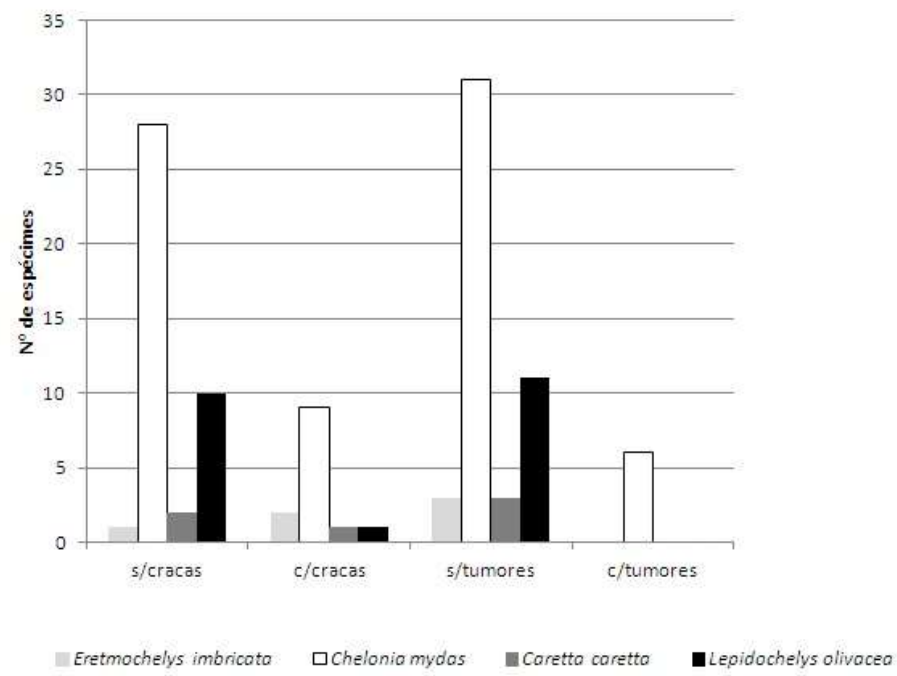

Figura 5: Representação de cracas distribuídas nas carapaças e a presença de tumores nas outras regiões do corpo das tartarugas encalhadas no litoral do Ipojuca/PE entre setembro/2015 a junho/2016.

\section{DISCUSSÃO}

Pesquisas realizadas por Scherer et al. (2014) no Rio Grande do Sul, monitoraram 4.320 km de praia e registraram 640 encalhes em três anos, como também resultados obtidos no Nordeste do Brasil, especificamente no litoral da Paraíba, com 124 encalhes entre agosto/ 2009 e julho/ 2010 em 15 km de praia (POLl et al., 2014). Tais resultados quando comparados com o número de encalhes para Pernambuco, entre 
os meses de setembro/ 2015 a junho/ 2016, obteve-se o menor registro de encalhes ( $n=54)$ em relação à Paraíba e ao Rio Grande do Sul. No entanto, vale destacar que a faixa monitorada em Ipojuca/PE, corresponde a apenas $12 \mathrm{~km}$ de praia dos $187 \mathrm{~km}$ de faixa litorânea. Além disso, os encalhes foram registrados mais efetivamente, durante as temporadas reprodutivas.

De um modo geral, fêmeas ficam mais vulneráveis aos encalhes, pois permanecem mais próximas a costa para desovar, tornando-se assim mais representativas em relação ao número em populações naturais, fato semelhante para o estado da Paraíba (POLI et al., 2014). Considera-se então que esse impacto foi mais relevante para as espécies C. mydas, L. olivacea e C. caretta, já que a espécie E. imbricata teve um contingente pequeno de indivíduos encalhados, esses resultados também são similares a outras localidades no Brasil, como Rio Grande Sul (BUGONI et al., 2001), São Paulo (SILVA et al., 2012) e Paraíba (POLI et al., 2014).

A espécie $C$. mydas, foi a que apresentou-se com maior registro de encalhe na região na forma jovem. De acordo com Hays et al. (2002), isso se deve principalmente porque essa espécie utiliza áreas neríticas para alimentação. Bjorndal et al. (1997) comentaram que a utilização de áreas neríticas acabam deixando esses animais mais suscetíveis a interações antrópicas o que possivelmente pode explicar o maior número de encalhes durante a fase juvenil. É importante ressaltar que essa espécie apesar de não ser a principal que desova na região, é a que tem sofrido um alto grau de impacto em todo litoral nordestino (RIBEIRO et al., 2014; SALES et al., 2007; MACEDO et al., 2011), existindo a necessidade de uma revisão pela IUCN e o ICMBio quanto ao status de conservação, pois ela tem demonstrado maior vulnerabilidade em termos de encalhes. De acordo com Bugoni et al. (2001), dentro dos $60 \%$ dos indivíduos encalhados foi possível observar uma alta ingestão de lixo antropogênico. Porém, esse trabalho não teve o foco em necropsia, sendo a observação apenas externa dos espécimes encalhados.

Quanto a variação dos intervalos de tamanhos dos juvenis e adultos foram obtidos resultados similares aos trabalhos de Goshe et al. (2010) para a espécie $C$. mydas, o qual registrou que os juvenis apresentaram uma média entre 50-59 cm e as fêmeas 89-101 cm de CCC. De acordo com Zug et al. (1986), a estimativa da idade de juvenis e adultos para a espécie $C$. caretta foi obtida através de médias de resultados de trabalhos realizados em diferentes localidades, os juvenis com comprimento curvilíneo da carapaça (<86 $\mathrm{cm}$ ) e os adultos $(>86 \mathrm{~cm})$, com valores próximos as fêmeas adultas obtidas neste trabalho. Segundo Zug et al. (2006), a espécie L. olivacea tem uma estimativa de idade para adultos (maturidade sexual) de $>60 \mathrm{~cm}$ de CCC e para juvenis (imaturos) com valores $<60 \mathrm{~cm}$ de CCC, sendo semelhante ao obtido no presente trabalho. Já para a espécie E. imbricata foi possível encontrar resultados similares com o trabalho realizado na Austrália em que os adultos tinham comprimentos da carapaça $\geq 80 \mathrm{~cm}$, e os juvenis (imaturos) $<80 \mathrm{~cm}$ (CHALOUPKA et al, 1997). De acordo com Guimarães et al. (2011), as fêmeas de tartarugas marinhas se aproximam da costa para a época de desova e os juvenis se aproximam principalmente para alimentação, por isso a maioria dos encalhes foram de fêmeas e juvenis das espécies citadas anteriormente.

A relação dos encalhes ocorrentes na região com os dados abióticos demostraram que em 2013 foi o maior número de encalhes, com destaque para o mês de fevereiro. Segundo Amarante et al. (2002) essa 
alta concentração de encalhes nos meses de janeiro, fevereiro, março, outubro e novembro pode ser explicado possivelmente pelas mudanças de direção dos ventos, que está relacionado também com a sazonalidade, alteração durante as estação, sendo o vento Nordeste, o de verão e o Sudeste, o de inverno. Sendo assim, o vento Nordeste sopra em direção à costa trazendo nutrientes e possivelmente aproximando os animais a região costeira, podendo aumentar a incidência de encalhes, trazendo carcaças de outros animais que morreram no mar para a costa, enquanto que o vento Sudeste faz o inverso.

Os meses menos chuvosos foram os mais representativos em relação ao número de encalhe, isso está provavelmente relacionado com a época em que há maior fluxo de embarcações de pesca (fevereiro, março, abril, setembro, novembro e dezembro), ou seja, meses de primavera e verão tiveram altos índices de encalhes em diferentes anos. Para a região Nordeste, as atividades de pesca aumentam nessa época, Silva et al. (2010) relataram a pesca de camarão no Estado de Sergipe, durante essa mesma época, como uma ameaça a conservação às tartarugas marinhas, já que esses animais estão migrando para suas áreas de nidificação. Poli et al. (2014) também comenta essa mesma problemática na costa do Estado da Paraíba, e além disso, tais animais podem realizar migrações para áreas de alimentação como o Arquipélago de Fernando de Noronha e Reserva Biológica Atol das Rocas sob influências sazonais, onde há uma variação de temperatura da água (MUSICK et al., 1997). Algumas pesquisas relatam que é comum C. mydas realizarem migrações para áreas de forrageio de zonas temperadas para latitudes maiores durante o verão, enquanto que os indivíduos que são de áreas tropicais se restringem a poucos movimentos (MUSICK et al., 1997). De uma maneira geral, observa-se (com.pess.) que um adensamento de barco nas áreas de Ipojuca na época de nidificação e concomitantemente uma melhor fiscalização.

\section{CONCLUSÕES}

Considerando o número de encalhes em relação a cada espécie, C. caretta $(n=15)$, E. imbricata $(n=24)$, L. olivacea $(n=82)$ e destacando C. mydas $(n=219)$, o presente trabalho obteve os resultados semelhantes aos encontrados de Poli et al. (2014), Scherer et al. (2014) e Silva et al. (2012). Bornatowski et al (2012), fizeram um levantamento dos registros de tartarugas encalhadas na Paraíba, e foram contabilizados 655 encalhes, os quais foram distribuídos para as espécies $C$. caretta $(n=5)$, L. olivacea $(n=10)$, E. imbricata $(\mathrm{n}=33)$, e $C$. mydas ( $\mathrm{n}=607)$, sendo esta última também com maiores números. Através de outros estudos é possível perceber que além da região do nordeste, a espécie $C$. mydas também tem maiores registros de encalhes em outras regiões da costa brasileira (SCHERER et al., 2014; SILVA et al., 2012; MACEDO et al., 2011), principalmente para região sul e sudeste, e umas das justificativas é seu hábito alimentar junto a costa (SILVA et al., 2012). Em relação ao número de tartarugas mortas e vivas, Bugoni et al. (2001) registraram 50 tartarugas encalhadas, na temporada de agosto de 1997 a julho de 1998 no litoral do Rio Grande do Sul, similar ao presente trabalho para as temporada de setembro de 2015 a junho de 2016.

De acordo com os registro de encalhes de tartarugas mortas e uma tartaruga viva de $C$. mydas, na temporada de setembro de 2015 a junho de 2016, a praia de Merepe foi a de maior incidência de encalhes, e de acordo com Moura et al. (2012) é a praia de maior extensão 3,47 km comparada as outras do litoral do 
Ipojuca, com menor declividade na sua geomorfologia. Segundo Júnior et al. (2011) a praia do Ipojuca possui uma corrente de deriva que passa na costa que tem direção de sul para norte na praia o que poderia explicar o maior número de encalhes na praia de Merepe, que está localizada mais ao norte, necessitando de ações conservacionistas nesse local.

Algumas tartarugas encalhadas observou-se a presença de cracas, um tipo de crustáceo encontrado em regiões bentônicas. Tal relação deve principalmente ao tempo que este animal esteve à deriva no mar, pois esta fauna (cracas) são animais oportunistas e podem aproveitar-se de animais debilitado ou morto como substrato para se fixar (BUGONI et al., 2001).Também foi observada a presença de tumores (lesões) compatíveis com a fibropapilomatose (FP) na espécie $C$. mydas. Essa doença tem acometido as tartarugas marinhas, principalmente a tartaruga verde (C. mydas) na região nordeste, segundo Poli et al. (2014) há ocorrência da doença em tartarugas verdes também na Paraíba. A presença de fibropapilomatose (FP) se deve principalmente pelas condições de saúde da tartaruga (imunossupressão), podendo causar sérios danos a mobilidade, comprometendo a sua alimentação podendo levar o animal à óbito (AGUIRRE et al., 1998).

Observou-se que $C$. mydas foi também à espécie com a maior quantidade de tumores, os quais foram registrados distribuídos nas regiões moles do corpo dos animais, como nadadeiras, pescoço e região dos olhos, resultandos os quais também foram obtidos por Mascarenhas et al. (2008) na Paraíba, com 74 indivíduos da espécie C. mydas de 278 encalhes. De acordo com Ene et al. (2005), sabe-se que a poluição marinha prejudica a saúde dos animais contribuindo para a ação do herpes vírus que causa a fibropapilomatose. Os resíduos antropogênicos podem ter sua origem através da população local e/ou por drenagem dos rios (GILLIGAN et al., 1992; LAIST et al., 1999), tais poluentes acabam ameaçando a vida marinha, principalmente das tartarugas marinhas que se aproximam da costa para se alimentar ou desovar e terminam ficando expostas a essa poluição (MASCARENHAS et al., 2008). O problema do lixo no mar é similar em todo litoral brasileiro, como também no litoral do município do Ipojuca, que tem uma das praias comum grande fluxo turístico, necessitando de políticas públicas mais eficientes em relação aos resíduos sólidos.

Algumas cidades brasileiras já utilizam do gerenciamento dos resíduos sólidos e a coleta seletiva como medidas de mitigação minimizando os impactos negativos causados pelo descarte destes resíduos no meio ambiente (CARVALHO et al., 2012). Sendo estas, as formas que podem fazer parte da gestão costeira, como uma política pública, junto com a educação ambiental realizada em cada temporada de forma continua para os municípios, em especial ao município do Ipojuca/PE.

\section{REFERÊNCIAS}

AGUIRRE, A. A.; SPRAKER, T. R; BALAZS, G. H.; ZIMMERMAN B.. Spirorchidiasis and fibropapillomatosis in green turtles from the hawaiian islands. Journal of Wildlife Diseases, Hawaii, v.34, n.1, p.91-98, 1998. Dol: http://doi.org/10.7589/0090-3558-34.1.91

AMARANTE, A. O. C.; SILVA, F. J. L.. Estado da Bahia: Atlas do potencial eólico. Projeto de Pesquisa e Desenvolvimento COELBA-ANEEL. 2002.
AYRES, M.; AYRES JÚNIOR, M.; AYRES, D. L.; SANTOS, A. D. A.. Aplicações estatísticas nas áreas das ciências biomédicas. Instituto Mamirauá, Belém, v.364, 2007.

BJORNDAL, K. A.. Nutritional ecology of sea turtles. Copeia, Miami, v.1985, n.3, p.736-751. 1985. DOI: http://doi.org/10.2307/1444767 
BJORNDAL, K. A.. Foraging ecology and nutrition of sea turtles. In: LUTZ P. L.; MUSICK, J. A.. The biology of sea turtles. p.199-231.1997.

BORNATOWSKI, H.; HEITHAUS, M. R.; BATISTA, C. M.; MASCARENHAS, R.. Shark scavenging and predation on sea turtles in northeastern Brazil. Amphibia-Reptilia, João Pessoa, v.33, n.3-4, p.495-502, 2012. DOI: http://doi.org/10.1163/15685381-00002852

BUGONI, L.; KRAUSE, L.; PETRY, M. V.. Marine debris and human impacts on sea turtles in southern Brazil. Marine pollution bulletin, Brasília, v.42, n.12, p.1330-1334, 2001. DOI: http://doi.org/10.1016/S0025-326X(01)00147-3

BUGONI, L.; KRAUSE, L.; ALMEIDA, A. O.; BUENO, A. A. P.. Commensal barnacles of sea turtles in Brazil. Marine Turtle Newsletter, v.94, p.7-9, 2001.

CAMACHO, M.; CALABUIG, P.; LUZARDO, O. P.; BOADA, L. D.; ZUMBADO, M.; ORÓS, J.. Crude oil as a stranding cause among loggerhead sea turtles (Caretta caretta) in the Canary Islands, Spain (1998-2011). Journal of wildlife diseases, v.49, n.3, p.637-640, 2013.

CARVALHO, A. C.; ABDALLAH, P. R.. Análise da gestão de resíduos sólidos no Terminal Porto Novo do Porto do Rio Grande, Brasil. Revista de Gestão Costeira Integrada, v.12, n.3, p.389-398, 2012.

CLUKEY, K. E.; LEPCZYKA, C. A.; BALAZS, G. H.; WORK, T. M. AND LYNCHE, J. M.. Investigation of plastic debris ingestion by four species of sea turtles collected as bycatch in pelagic Pacific longline fisheries. Marine Pollution Bulletin, v.120, n.1-2, p.117-125, 2017. DOI:

http://doi.org/10.1016/j.marpolbul.2017.04.064

ENE, A.; SU, M.; LEMAIRE, S.; ROSE, C.; SCHAFF, S.; MORETTI, R.; HERBST, L. H.. Distribution of chelonid fibropapillomatosis-associated herpesvirus variants in Florida: molecular genetic evidence for infection of turtles following recruitment to neritic developmental habitats. Journal of Wildlife Diseases, v.41, n.3, p.489-497. 2005. DOI: http://doi.org/10.7589/0090-3558-41.3.489

EPPERLY, S. P.; BRAUN, J.; CHESTER, A. J.; CROSS, F. A.; MERRINER, J. V.; TESTER, P. A.; CHURCHILL, J. H.. Beach strandings as an indicator of at-sea mortality of sea turtles. Bulletin of Marine Science, Miami, v.59, n.2, p.289297, 1996.

HAYS, G. C.; BRODERICK, A. C.; GODLEY, B. J.; LOVELL, P.; MARTIN, C.; MCCONNELL, B. J.; RICHARDSON, S.. Biphasal long-distance migration in green turtles. Animal Behaviour, Brasília, v.64, n.6, p.895-898, 2002. DOI: http://doi.org/10.1006/anbe.2002.1975

GILLIGAN, M. R.; PITTS, R. S.; RICHARDSON, J. P.; KOZEL, T. R.. Rates of Accumulation of Marine Debris in Chatham County, Georgia. Marine Pollution Bulletin, Tiblissi, v.24, n.9, p.436-441, 1992.

GUIMARÃES, E. S.; MOURA, G. J. B.; LINS, E. A. M.. Aspectos Ecológicos de Eretmochelys imbricata entre os anos $2000 \mathrm{e}$ 2008 nas praias de Ipojuca-PE e lista comentada das espécies de tartarugas marinhas ocorrentes no Estado de Pernambuco. In: MOURA, G. J. B.; SANTOS, E. M.; OLIVEIRA,
M. A. B.; CABRAL, M. C. C.. Herpetologia do Estado de Pernambuco. Brasília: Ministério do Meio Ambiente, 2010. p.305-317.

IUCN. International Union for Conservation of Nature. Red List of Threatened Species. version 2018-2. 2018.

JUNIOR, J. L. B.; DIAZ, X. G.; NEUMANN-LEITÃO, S.. Diversidade de larvas de peixes das áreas internas e externas do porto de Suape (Pernambuco-Brazil). Tropical Oceanography, v.39, p.1-13, 2011.

LAIST, D. W.; COE, J. M.; O'HARA, K. J.. Marine Debris Pollution. In: TWISS JR, J. R.; REEVES, R. R.. Conservation and management of marine mammals. Smithsonian Institution Press. 1999.

MASCARENHAS, R.; BATISTA, C. P.; MOURA, I. F.; CALDAS, A. R.; NETO, J. M. C.; VASCONCELOS, M. Q.; ROSA, S. S.; BARROS, T. V. S. Lixo marinho em área de reprodução de tartarugas marinhas no estado da Paraíba (Nordeste do Brasil). Revista de Gestão Costeira Integrada-Journal of Integrated Coastal Zone Management, João Pessoa, v.8, n.2, 2008.

MASCARENHAS, R.; IVERSON, P. J.. Fibropapillomatosis in Stranded Green Turtles (Chelonia mydas) in Paraiba State, Northeastern Brazil: Evidence of a Brazilian Epizootic?. Marine Turtle Newsletter, João Pessoa, v.120, p.3-6, 2008.

MASCARENHAS, R.; SANTOS, R.; ZEPPELINI, D.. Stranded sea turtles on the coast of Paraiba, Brazil. Marine Turtle Newsletter, João Pessoa, v.107, p.13-14, 2005.

MARCOVALDI, M. A.; SANTOS, A. S.; SALES, G.. Plano de Ação Nacional para Conservação das Tartarugas marinhas. Brasília: ICMBio, 2011.

MOURA, C. C. D. M.; GUIMARÃES, E. D. S.; MOURA, G. J. B.; AMARAL, G. A.; SILVA, A. C.. Distribuição espaço-temporal e sucesso reprodutivo de Eretmochelys imbricata nas praias do Ipojuca, Pernambuco, Brasil. Iheringia série Zoologia, Recife, v.102, n.3, p.254-260, 2012.

MUSICK, J. A.; LIMPUS, C. J.. Habitat utilization and migration in juvenile sea turtles. In: LUTZ, P.; MUSICK, J. A.. The biology of sea turtles. Tallahassee: CRC Press, 1997. p.151178.

POLI, C.; LOPEZ, L. C. S.; MESQUITA, D. O.; SASKA, C.; MASCARENHAS, R.. Patterns and inferred processes associated with sea turtle strandings in Paraíba State, Northeast Brazil. Brazilian Journal of Biology, João Pessoa, v.74, n.2, p.283-289, 2014. DOI: http://dx.doi.org/10.1590/1519-6984.13112

RIBEIRO, A. B. N.; BARRETO, L.; RIBEIRO, L. E. S.; AZEVEDO, R. R.. Conservation aspects of sea turtles in Maranhao island, Sao Luis, Brazil. Bioscience Journal, São Luís, v.30, n.3, 2014.

RODAMILANS, M. G.; TORRES, P. T.; ROSTÁN, G.; WROBEL, G. D.; CUSTÓDIO, L. D.; GARCEZ, N. A. F.; FRANKE, C. R. . Ingestão de resíduos antropogênicos por tartarugas marinhas no litoral norte do estado da Bahia, Brasil. Ciência Rural, Salvador, v.41, n.11, 2011. 
SALES, G.; GIFFONI, B. B.; BARATA, P. C.. Incidental catch of sea turtles by the Brazilian pelagic longline fishery. Journal of the Marine Biological Association of the United Kingdom, Brasília, v.88, n.4, p.853-864, 2008. DOI: http://doi.org/10.1017/S0025315408000441

STATSOFT I. Statistica: Quick Reference. 2013.

SANCHES, T. M.; BELLINI, C.. Juvenile Eretmochelys imbricata and Chelonia mydas in the Archipelago of Fernando de Noronha, Brazil. Chelonian Conservation and Biology, Recife, v.3, n.2, p.308-311, 1999.

SCHERER, A. L.; VALLS, F. C. L.; BASLER, A. B.; SCHERER, J. M. F.; PETRY, M. V.. Life Stages, Anthropogenic Impact, and Temporal Variation of Stranded Sea Turtles in Southern Brazil. Chelonian Conservation and Biology, v.13, n.1, p.4248, 2014. DOI: http://doi.org/10.2744/CCB-1029.1

SILVA, C. C.; VARELA JR, A. S.; BARCAROLLI, I. F.; BIANCHINI, A.. Concentrations and distributions of metals in tissues of stranded green sea turtles (Chelonia mydas) from the southern Atlantic coast of Brazil. Science of the Total Environment, v.466, p.109-118, 2014. DOI: http://doi.org/10.1016/i.scitotenv.2013.06.094

SILVA, A. C. C. D.; CASTILHOS, J. C.; SANTOS, E. A. P.; BRONDÍZIO, L. S.; BUGONI, L.. Efforts to reduce sea turtle bycatch in the shrimp fishery in Northeastern Brazil through a co-management process. Ocean \& Coastal Management,
Aracaju, v.53, n.9, p.570-576, 2010. DOI: http://doi.org/10.1016/i.ocecoaman.2010.06.016

SILVA, G. C.; VAZ-DOS-SANTOS, A. M.; MARACINI, P.. Análise De Encalhes De Tartarugas Marinhas (Testudines: Cheloniidae e Dermochelydae) nos Municípios Da Baixada Santista , Iguape e Cananéia no período de 2004. Revista Ceciliana, São Paulo, v.4, n.2, p.9-15, 2012.

SALES, G.; LOPEZ, G. G.; SANTOS, A. S.; VIANNA, P.; SERAFINI, T. Z.. Captura incidental de tartarugas marinhas na pesca artesanal registrada pelo projeto TAMAR-IBAMA no litoral norte da Bahia, Brasil. In: CONGRESSO LATINO AMERICANO DE CIÊNCIAS DO MAR, 12. Anais. Florianópolis, 2007.

VÉLEZ-RUBIO, G. M.; ESTRADES, A.; FALLABRINO, A.; TOMÁS, J.. Marine turtle threats in Uruguayan waters: insights from 12 years of stranding data. Marine Biology, Montevidéu, v.160, n.11, p.2797-2811, 2013.DOI: http://doi.org/10.1007/s00227-013-2272-y

WYNEKEN, J.; BURKE, T. J.; SALMON, M.; PEDERSEN, D. K.. Egg failure in natural and relocated sea turtle nests. Journal of Herpetology, Estados Unidos da América, v.22, n.1, p.8896, 1988.

WYNEKEN, J.; WITHERINGTON, D.. The anatomy of sea turtles. U.S. Department of commerce NOAA Technical Memorandum NMFS-SEFSC-470. 2001.

A CBPC - Companhia Brasileira de Produção Científica (CNPJ: 11.221.422/0001-03) detém os direitos materiais desta publicação. Os direitos referem-se à publicação do trabalho em qualquer parte do mundo, incluindo os direitos às renovações, expansões e disseminações da contribuição, bem como outros direitos subsidiários. Todos os trabalhos publicados eletronicamente poderão posteriormente ser publicados em coletâneas impressas sob coordenação da Sustenere Publishing, da Companhia Brasileira de Produção Científica e seus parceiros autorizados. Os (as) autores (as) preservam os direitos autorais, mas não têm permissão para a publicação da contribuição em outro meio, impresso ou digital, em português ou em tradução. 